\title{
Bifurcation and chaos control in a discrete-time predator-prey model with nonlinear saturated incidence rate and parasite interaction
}

\author{
Waqas Ishaque ${ }^{1,2}$, Qamar Din ${ }^{2 *}$, Muhammad Taj ${ }^{1}$ and Muhammad Asad Iqbal ${ }^{2}$
}

\section{"Correspondence:}

qamar.sms@gmail.com

${ }^{2}$ Department of Mathematics,

University of Poonch Rawalakot,

Azad Kashmir, Pakistan

Full list of author information is

available at the end of the article

\section{Springer}

\begin{abstract}
The dynamical behavior of the predator-prey system is influenced effectively due to the mutual interaction of parasites. Regulations are imposed on biodiversity due to such type of interaction. With implementation of nonlinear saturated incidence rate and piecewise constant argument method of differential equations, a three-dimensional discrete-time model of prey-predator-parasite type is studied. The existence of equilibria and the local asymptotic stability of these steady states are investigated. Moreover, explicit criteria for a Neimark-Sacker bifurcation and a period-doubling bifurcation are implemented at positive equilibrium point of the discrete-time model. Chaos control is discussed through implementation of a hybrid control technique based on both parameter perturbation and a state feedback strategy. At the end, some numerical simulations are provided to illustrate our theoretical discussion.
\end{abstract}

Keywords: Eco-epidemiological model; Local stability; Hopf bifurcation; Flip bifurcation; Chaos control

\section{Introduction}

Trophic interactions, keystone species, food webs, competition and biodiversity are greatly affected due to interaction of parasites and consequently the community structure can be reverted. Furthermore, qualitative analysis of prey and predator interaction is a distinguished field for investigation in mathematical ecology. On the other hand, presence of some kind of disease in prey-predator interaction is a prominent topic for investigation, and this is a comparatively new subject in the field of eco-epidemiology. In order to observe the spread of some sort of disease, both aspects for ecology and epidemiology are included in such a field of study. It is important to point out some earlier studies in the area related to mathematical ecology and epidemiology. For this purpose, Hadeler and Freedman [1] studied a prey-predator system in which both types of species (prey and predator) were subjected to parasitism. Freedman [2] investigated a prey-predator model in which the predator population is always treated as primary host whereas the prey population density may be either a secondary host or a primary host for the parasite interaction. Beltrami and Carroll [3] studied a simple mathematical system of trophic

(c) The Author(s) 2019. This article is distributed under the terms of the Creative Commons Attribution 4.0 International License (http://creativecommons.org/licenses/by/4.0/), which permits unrestricted use, distribution, and reproduction in any medium, provided you give appropriate credit to the original author(s) and the source, provide a link to the Creative Commons license, and indicate if changes were made. 
type in which a mortality of virus-induced type is included, and the actual bloom patterns for several species are also presented. Venturino [4] investigated prey-predator systems subjected to some sort of disease in prey density. Moreover, Chattopadhyay and Arino [5] examined a three species eco-epidemiological model, that is, the predator population, the infected prey, and the sound prey. Keeping in view the interaction of three species for an eco-epidemiological model consisting of predator density, infected prey and sound prey, a mathematical model was investigated by Chattopadhyay et al. [6]. Furthermore, a Hopf bifurcation was also studied at its positive steady state.

Next, keeping in view an eco-epidemiological model of pelicans at risk in the Salton Sea studied by Chattopadhyay et al. [7], we consider the following predator-prey-parasite model:

$$
\left\{\begin{array}{l}
\frac{d x}{d t}=s x\left(1-\frac{x+y}{k}\right)-\alpha x y, \\
\frac{d y}{d t}=\alpha x y-\frac{\beta y z}{y+\delta}-\mu y, \\
\frac{d z}{d t}=\frac{\beta y y z}{y+\delta}-\eta z,
\end{array}\right.
$$

where $x(t), y(t)$, and $z(t)$ denote densities of susceptible Tilapia fish population, infected Tilapia fish population and predator population known as Pelican birds, respectively. Moreover, $x$ and $y$ are the state variables to represent the susceptible prey population density and infected prey population density, respectively, at time $t$, and $z$ represents predator population density at time $t$. Furthermore, it is supposed that the population for prey obeys the logistic growth function where $s$ is taken as the growth rate and $k$ represents the carrying capacity. Moreover, $\alpha$ denotes the coefficient for disease transmission, $\mu$ represents the death rate of the infected Tilapia fish population, $\delta$ is a constant for half-saturation rate, $\beta$ is the capturing capacity of predators, $\gamma$ is used for the conversion efficiency of predator, and $\eta$ represents the food-dependent death rate related to the predator population. For more investigations and modifications related to system (1.1) we refer to [812]. All these modifications and discussions are implemented to continuous counterparts of system (1.1). Hence it is worthwhile to discuss discrete counterparts of system (1.1) in which populations are treated as generations of non-overlapping type. Furthermore, there are a lot of mathematical investigations related to flip bifurcation, Hopf bifurcation and controlling chaos in planar discrete-time models and a little work is performed for controlling chaos and bifurcation analysis for discrete-time systems of three or more dimensions. Hence, it is worthwhile to discuss the bifurcation analysis and controlling chaos in the case of a three-dimensional discrete-time systems.

By replacing the bilinear incidence rate in the saturated incidence rate of nonlinear type the following modification is proposed for the system (1.1):

$$
\left\{\begin{array}{l}
\frac{d x}{d t}=s x\left(1-\frac{x+y}{k}\right)-\frac{\alpha x y}{1+c y}, \\
\frac{d y}{d t}=\frac{\alpha x y}{1+c y}-\frac{\beta y z}{y+\delta}-\mu y, \\
\frac{d z}{d t}=\frac{\beta \gamma y z}{y+\delta}-\eta z,
\end{array}\right.
$$

where $c$ denotes the contact rate constant for the crowding effect. Moreover, system (1.2) shows that other fish cannot be caught by Pelicans birds, besides Tilapia. This fact is compatible with natural phenomena because in the literature there are several experimental 
studies in favor of this argument. Moreover, since system (1.2) is a modification of an ecoepidemiological model of Pelicans at risk in the Salton Sea by Chattopadhyay et al. [7], and it is pointed out in [13] that very few fish species can endure living in the Salton Sea due to the high salinity. Due to the presence of highly salty and polluted water, only Tilapia fish have survival tendency in Salton Sea. Consequently, other species of freshwater fish cannot survive in salty water.

According to survey reports published by US Geological Survey National Wildlife Health Center from 1978 to 2003 many white pelicans were to die out due to type $C$ botulism [14]. In 1996, over 8500 white pelicans had died at Salton Sea in California due to infection of type $C$ botulism. The major cause of that disaster were Tilapia fish which are a key source for $C$ botulism toxins produced in white pelican birds. In addition, the study has indicated that bacterial infections take part in lowering oxygen levels in the tissues of affected fish. Due to lack of oxygen, the fish find oxygen from the ocean surface and this leads to an appropriate environment to produce botulism in the tissues of the infected fish. When pelicans attack on these defenseless fish, the botulism toxins is ingested by pelican birds and as a result avian botulism is produced in their bodies [15].

Moreover, in system (1.2), it is assumed that pelican birds only prey infected Tilapia, but do not capture the healthy Tilapia because the infected fish are feeble and become easier to prey. On the other hand, there is a significant number of infected Tilapia fish present in the Salton Sea and due to their struggle against death these are more unprotected and attractive to the Pelican birds [7]. Meanwhile, healthy Tilapia easily escape and predation becomes difficult.

For qualitative analysis, including local stability, bifurcations analysis and chaos control for a discrete counterpart of system (1.2), a piecewise constant argument is implemented to obtain the following exponential form of nonlinear difference equations:

$$
\left\{\begin{array}{l}
x_{n+1}=x_{n} \exp \left(s\left(1-\frac{x_{n}+y_{n}}{k}\right)-\frac{\alpha y_{n}}{1+c y_{n}}\right), \\
y_{n+1}=y_{n} \exp \left(\frac{\alpha x_{n}}{1+c y_{n}}-\frac{\beta z_{n}}{y_{n}+\delta}-\mu\right), \\
z_{n+1}=z_{n} \exp \left(\frac{\beta \gamma y_{n}}{y_{n}+\delta}-\eta\right) .
\end{array}\right.
$$

Moreover, the remaining discussion for this paper is organized as follows. In Sect. 2, we explore the existence of equilibria and conditions for their local asymptotic stability. In Sect. 3, an explicit criterion for Hopf bifurcation is implemented at positive steady state of system (1.3). Section 4 is dedicated to an implementation of an explicit criterion for flip bifurcation at a positive equilibrium of system (1.3). In Sect. 5, a chaos control strategy based on parameter perturbation and a state feedback control methodology is implemented to system (1.3). Finally, some suitable numerical simulations are presented in Sect. 6 for verification of our theoretical discussion.

\section{Existence of equilibria and stability}

We investigate steady states and criteria for local asymptotic stability of these equilibria. For this purpose, we suppose that $\mu<k \alpha$, then we obtain the following trivial and boundary steady states for model (1.3):

$$
E_{0}=(0,0,0), \quad E_{1}=(k, 0,0), \quad E_{2}=\left(u^{*}, v^{*}, 0\right),
$$


where

$$
u^{*}:=\frac{\mu(s+c k s-k \alpha)+\mu \sqrt{(s+c k s)^{2}+k^{2} \alpha^{2}+2 k s(\alpha-c k \alpha+2 c \mu)}}{2 s(\alpha+c \mu)}
$$

and

$$
v^{*}:=\frac{\alpha(c k s-s-k \alpha)-2 c s \mu+\alpha \sqrt{(s+c k s)^{2}+k^{2} \alpha^{2}+2 k s(\alpha-c k \alpha+2 c \mu)}}{2 c s(\alpha+c \mu)} .
$$

Furthermore, there exists a unique positive steady state for system (1.3) if the following conditions are satisfied:

$$
\left\{\begin{array}{l}
s \delta \eta(\eta-\beta \gamma-c \delta \eta)+k(\beta \gamma-\eta)(s \beta \gamma-\eta(s-c s \delta+\alpha \delta))>0 \\
\beta \gamma>\eta \\
k \alpha(\beta \gamma-\eta)(s \beta \gamma-\eta(s-c s \delta+\alpha \delta)) \\
\quad>s(\beta \gamma+\eta(c \delta-1))(\alpha \delta \eta+\mu(\beta \gamma-\eta+c \delta \eta)) .
\end{array}\right.
$$

Assume that (2.1) holds true, then positive steady state for model (1.3) is written as $E_{*}=$ $\left(x^{*}, y^{*}, z^{*}\right)$, where

$$
x^{*}:=\frac{s \delta \eta(\eta-\beta \gamma-c \delta \eta)+k(\beta \gamma-\eta)(s \beta \gamma-\eta(s-c s \delta+\alpha \delta))}{s(\beta \gamma-\eta)(\beta \gamma+\eta(c \delta-1))}, \quad y^{*}:=\frac{\eta \delta}{\beta \gamma-\eta},
$$

and

$$
\begin{aligned}
z^{*}:= & (\gamma \delta(k \alpha(\beta \gamma-\eta)(s \beta \gamma-\eta(s-c s \delta+\alpha \delta)) \\
& -s(\beta \gamma+\eta(c \delta-1))(\alpha \delta \eta+\mu(\beta \gamma-\eta+c \delta \eta)))) \\
& /\left(s(\beta \gamma-\eta)(\beta \gamma+\eta(c \delta-1))^{2}\right) .
\end{aligned}
$$

Keeping in view the local asymptotic stability for three-dimensional systems of discrete type, the following lemma is presented.

Lemma 2.1 ([16]) We consider a cubic polynomial equation of the following type:

$$
\lambda^{3}+\alpha_{2} \lambda^{2}+\alpha_{1} \lambda+\alpha_{0}=0
$$

where $\alpha_{2}, \alpha_{1}$ and $\alpha_{0}$ represent real constants. Furthermore, all roots for the polynomial Eq. (2.2) lie within the open unit disk if and only if the following conditions are satisfied:

$$
\left|\alpha_{2}+\alpha_{0}\right|<1+\alpha_{1}, \quad\left|\alpha_{2}-3 \alpha_{0}\right|<3-\alpha_{1}, \quad \text { and } \quad \alpha_{0}^{2}+\alpha_{1}-\alpha_{0} \alpha_{2}<1 .
$$

Furthermore, the Jacobian matrix $J_{(1.3)}\left(E_{0}\right)$ for system (1.3) evaluated at the trivial equilibrium $E_{0}$ is given by

$$
J_{(1.3)}\left(E_{0}\right)=\left[\begin{array}{ccc}
\exp (s) & 0 & 0 \\
0 & \exp (-\mu) & 0 \\
0 & 0 & \exp (-\eta)
\end{array}\right] .
$$


Then it follows from $J_{(1.3)}\left(E_{0}\right)$ that $E_{0}$ is an unstable steady state for system (1.3). Moreover, the variational matrices computed about $E_{1}$ and $E_{2}$ are given by

$$
J_{(1.3)}\left(E_{1}\right)=\left[\begin{array}{ccc}
1-s & -\alpha k-s & 0 \\
0 & e^{k \alpha-\mu} & 0 \\
0 & 0 & e^{-\eta}
\end{array}\right]
$$

and

$$
J_{(1.3)}\left(E_{2}\right)=\left[\begin{array}{ccc}
1-\frac{s u^{*}}{k} & \frac{u^{*}\left(s+k \alpha+c s v^{*}\left(2+c v^{*}\right)\right)}{k\left(1+c v^{*}\right)^{2}} & 0 \\
\frac{\alpha v^{*}}{1+c v^{*}} & \frac{1+c v^{*}\left(2-\alpha u^{*}+c v^{*}\right)}{\left(1+c v^{*}\right)^{2}} & -\frac{\beta v^{*}}{\delta+v^{*}} \\
0 & 0 & e^{\frac{\beta \gamma v^{*}}{\delta+v^{*}}-\eta}
\end{array}\right] .
$$

Furthermore, the characteristic polynomial equation for $J_{(1.3)}\left(E_{2}\right)$ is calculated as follows:

$$
P_{1}(\lambda)=\lambda^{3}+\beta_{2} \lambda^{2}+\beta_{1} \lambda+\beta_{0}=0
$$

where

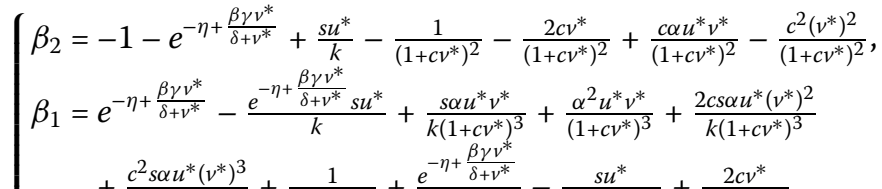

$$
\begin{aligned}
& +\frac{c^{2} s \alpha u^{*}\left(v^{*}\right)^{3}}{k\left(1+c v^{*}\right)^{3}}+\frac{1}{\left(1+c v^{*}\right)^{2}}+\frac{e^{-\eta+\frac{\beta}{\delta+v^{*}}}}{\left(1+c v^{*}\right)^{2}}-\frac{s u^{*}}{k\left(1+c v^{*}\right)^{2}}+\frac{2 c v^{*}}{\left(1+c v^{*}\right)^{2}} \\
& +\frac{2 c e^{-\eta+\frac{\beta \gamma v^{*}}{\delta+v^{*}}} v^{*}}{\left(1+c v^{*}\right)^{2}}-\frac{2 c s u^{*} v^{*}}{k\left(1+c v^{*}\right)^{2}}-\frac{c \alpha u^{*} v^{*}}{\left(1+c v^{*}\right)^{2}}-\frac{c e^{-\eta+\frac{\beta \gamma v^{*}}{\delta+v^{*}} \alpha u^{*} v^{*}}}{\left(1+c v^{*}\right)^{2}}+\frac{c s \alpha\left(u^{*}\right)^{2} v^{*}}{k\left(1+c v^{*}\right)^{2}} \\
& +\frac{c^{2}\left(v^{*}\right)^{2}}{\left(1+c v^{*}\right)^{2}}+\frac{c^{2} e^{-\eta+\frac{\beta \gamma v^{*}}{\delta+v^{*}}}\left(v^{*}\right)^{2}}{\left(1+c v^{*}\right)^{2}}-\frac{c^{2} s u^{*}\left(v^{*}\right)^{2}}{k\left(1+c v^{*}\right)^{2}}, \\
& \beta_{0}=-\frac{e^{-\eta+\frac{\beta \gamma v^{*}}{\delta+v^{*}}} s \alpha u^{*} v^{*}}{k\left(1+c v^{*}\right)^{3}}-\frac{e^{-\eta+\frac{\beta \gamma v^{*}}{\delta+v^{*}}} \alpha^{2} u^{*} v^{*}}{\left(1+c v^{*}\right)^{3}}-\frac{2 c e^{-\eta+\frac{\beta \gamma v^{*}}{\delta+v^{*}}} s \alpha u^{*}\left(v^{*}\right)^{2}}{k\left(1+c v^{*}\right)^{3}}-\frac{e^{-\eta+\frac{\beta \gamma v^{*}}{\delta+v^{*}}}}{\left(1+c v^{*}\right)^{2}} \\
& -\frac{c^{2} e^{-\eta+\frac{\beta \gamma v^{*}}{\delta+v^{*}}} s \alpha u^{*}\left(v^{*}\right)^{3}}{k\left(1+c v^{*}\right)^{3}}+\frac{e^{-\eta+\frac{\beta \gamma v^{*}}{\delta+v^{*}}} s u^{*}}{k\left(1+c v^{*}\right)^{2}}-\frac{2 c e^{-\eta+\frac{\beta \gamma v^{*}}{\delta+v^{*}}} v^{*}}{\left(1+c v^{*}\right)^{2}}+\frac{2 c e^{-\eta+\frac{\beta \gamma v^{*}}{\delta+v^{*}}} s u^{*} v^{*}}{k\left(1+c v^{*}\right)^{2}} \\
& +\frac{c e^{-\eta+\frac{\beta \gamma v^{*}}{\delta+v^{*}}} \alpha u^{*} v^{*}}{\left(1+c v^{*}\right)^{2}}-\frac{c e^{-\eta+\frac{\beta \gamma v^{*}}{\delta+v^{*}}} s \alpha\left(u^{*}\right)^{2} v^{*}}{k\left(1+c v^{*}\right)^{2}}-\frac{c^{2} e^{-\eta+\frac{\beta \gamma v^{*}}{\delta+v^{*}}}\left(v^{*}\right)^{2}}{\left(1+c v^{*}\right)^{2}}+\frac{c^{2} e^{-\eta+\frac{\beta \gamma v^{*}}{\delta+v^{*}}} s u^{*}\left(v^{*}\right)^{2}}{k\left(1+c v^{*}\right)^{2}} .
\end{aligned}
$$

Furthermore, the local asymptotic behavior for steady states $E_{1}$ and $E_{2}$ of the system (1.3) is given in the following lemma.

\section{Lemma 2.2}

(i) Equilibrium point $E_{1}$ for model (1.3) is a sink and locally asymptotically stable if and only if the following conditions are satisfied:

$$
s<2, \quad k \alpha<\mu .
$$

(ii) Suppose that $\mu<k \alpha$, then the equilibrium point $E_{2}$ for model (1.3) is a sink and locally asymptotically stable if and only if the following conditions are satisfied:

$$
\left|\beta_{2}+\beta_{0}\right|<1+\beta_{1}, \quad\left|\beta_{2}-3 \beta_{0}\right|<3-\beta_{1}, \quad \text { and } \quad \beta_{0}^{2}+\beta_{1}-\beta_{0} \beta_{2}<1,
$$

where $\beta_{2}, \beta_{1}$ and $\beta_{0}$ are written in (2.4). 
Furthermore, the Jacobian matrix denoted by $J_{(1.3)}\left(E_{*}\right)$ for system (1.3) evaluated about the equilibrium point $E_{*}$ is calculated as follows:

$$
J_{(1.3)}\left(E_{*}\right)=\left(\begin{array}{ccc}
1-\frac{s x^{*}}{k} & a_{12} & 0 \\
\frac{\alpha \delta \eta}{\beta \gamma-\eta+c \delta \eta} & 1+a_{22} & -\frac{\eta}{\gamma} \\
0 & a_{32} & 1
\end{array}\right),
$$

where

$$
\left\{\begin{array}{l}
a_{12}=-x^{*}\left(\frac{s}{k}+\frac{\alpha}{\left(1+c y^{*}\right)^{2}}\right), \\
a_{22}=\frac{\beta y^{*} z^{*}}{\left(\delta+y^{*}\right)^{2}}-\frac{c \alpha x^{*} y^{*}}{\left(1+c y^{*}\right)^{2}}, \\
a_{32}=\frac{\beta \gamma \delta z^{*}}{\left(\delta+y^{*}\right)^{2}} .
\end{array}\right.
$$

Next, the characteristic polynomial equation for $J_{(1.3)}\left(E_{*}\right)$ is given by

$$
P_{1}(\lambda)=\lambda^{3}+\gamma_{2} \lambda^{2}+\gamma_{1} \lambda+\gamma_{0}=0
$$

where

$$
\begin{aligned}
& \left\{\begin{array}{l}
\gamma_{2}=-3+\frac{s x^{*}}{k}+\frac{c \alpha x^{*} y^{*}}{\left(1+c y^{*}\right)^{2}}-\frac{\beta y^{*} z^{*}}{\left(\delta+y^{*}\right)^{2}}, \\
\gamma_{1}=3-\frac{2 s x^{*}}{k}+\frac{s \delta \eta x^{*}}{k(\beta \gamma-\eta+c \delta \eta)\left(1+c y^{*}\right)^{2}}+\frac{\alpha^{2} \delta \eta x^{*}}{(\beta \gamma-\eta+c \delta \eta)\left(1+c y^{*}\right)^{2}} \\
\quad 2 c \alpha x^{*} y^{*}+\frac{2 c s \alpha \delta \eta x^{*} y^{*}}{\left(1+y \alpha\left(x^{*}\right)^{2} \gamma^{*}\right.}
\end{array}\right. \\
& -\frac{2 c \alpha x^{*} y^{*}}{\left(1+c y^{*}\right)^{2}}+\frac{2 c s \alpha \delta \eta x^{*} y^{*}}{k(\beta \gamma-\eta+c \delta \eta)\left(1+c y^{*}\right)^{2}}+\frac{c s \alpha\left(x^{*}\right)^{2} y^{*}}{k\left(1+c y^{*}\right)^{2}} \\
& +\frac{c^{2} s \alpha \delta \eta x^{*}\left(y^{*}\right)^{2}}{k(\beta \gamma-\eta+c \delta \eta)\left(1+c y^{*}\right)^{2}}+\frac{\beta \delta \eta z^{*}}{\left(\delta+y^{*}\right)^{2}}+\frac{2 \beta y^{*} z^{*}}{\left(\delta+y^{*}\right)^{2}}-\frac{s \beta x^{*} y^{*} z^{*}}{k\left(\delta+y^{*}\right)^{2}}, \\
& \gamma_{0}=-1+\frac{s x^{*}}{k}-\frac{s \alpha \delta \eta x^{*}}{k(\beta \gamma-\eta+c \delta \eta)\left(1+c y^{*}\right)^{2}}-\frac{\alpha^{2} \delta \eta x^{*}}{(\beta \gamma-\eta+c \delta \eta)\left(1+c y^{*}\right)^{2}} \\
& +\frac{c \alpha x^{*} y^{*}}{\left(1+c y^{*}\right)^{2}}-\frac{2 c s \delta \delta \eta x^{*} y^{*}}{k(\beta \gamma-\eta+c \delta \eta)\left(1+c y^{*}\right)^{2}}-\frac{c s \alpha\left(x^{*}\right)^{2} y^{*}}{k\left(1+c y^{*}\right)^{2}} \\
& -\frac{c^{2} s \alpha \delta \eta x^{*}\left(y^{*}\right)^{2}}{k(\beta \gamma-\eta+c \delta \eta)\left(1+c y^{*}\right)^{2}}-\frac{\beta \delta \eta z^{*}}{\left(\delta+y^{*}\right)^{2}}+\frac{s \beta \delta \eta x^{*} z^{*}}{k\left(\delta+y^{*}\right)^{2}}-\frac{\beta y^{*} z^{*}}{\left(\delta+y^{*}\right)^{2}}+\frac{s \beta x^{*} y^{*} z^{*}}{k\left(\delta+y^{*}\right)^{2}} .
\end{aligned}
$$

One can implement Lemma 2.1 to investigate the stability conditions for $E^{*}$ as follows.

Lemma 2.3 Suppose that the conditions given in (2.1) are satisfied, then the steady state $E_{*}$ for model (1.3) is a sink and it is locally asymptotically stable if and only if the following inequalities are satisfied:

$$
\left|\gamma_{2}+\gamma_{0}\right|<1+\gamma_{1}, \quad\left|\gamma_{2}-3 \gamma_{0}\right|<3-\gamma_{1}, \quad \text { and } \quad \gamma_{0}^{2}+\gamma_{1}-\gamma_{0} \gamma_{2}<1
$$

where $\gamma_{2}, \gamma_{1}$ and $\gamma_{0}$ are written in (2.7).

\section{Hopf bifurcation analysis}

Here we investigate the parametric conditions for which the positive steady state for discrete-time system (1.3) undergoes a Neimark-Sacker (Hopf) bifurcation. For such an investigation an explicit criterion of Hopf bifurcation is used without computing the eigenvalues for the variational matrix of system under consideration (see also [17, 18]). For this purpose, an explicit criterion for Hopf bifurcation is given below. 
Lemma 3.1 ([19]) An n-dimensional system of discrete type is considered as follows:

$$
Z_{k+1}=F_{\xi}\left(Z_{k}\right)
$$

where $\xi \in \mathbb{R}$ is used for bifurcation parameter. Furthermore, considering characteristic polynomial of the Jacobian matrix $J\left(Z^{*}\right)=\left(\theta_{i j}\right)_{n \times n}$ evaluated at fixed point $Z^{*} \in \mathbb{R}^{n}$ for $F_{\xi}$ as follows:

$$
P_{\xi}(\lambda)=\lambda^{n}+\tau_{1} \lambda^{n-1}+\cdots+\tau_{n-1} \lambda+\tau_{n}
$$

where $\tau_{i}=\tau_{i}(\xi, u), i=1,2, \ldots, n$, and $u$ is referred to as the controlling parameter, or it may be some other parameter which is to be determined. Next, we take into account a sequence of the form $\left(\mathbb{D}_{i}^{ \pm}(\xi, u)\right)_{i=0}^{n}$ of determinants such that $\mathbb{D}_{0}^{ \pm}(\xi, u)=1$, and it is defined as follows:

$$
\mathbb{D}_{i}^{ \pm}(\xi, u)=\operatorname{det}\left(T_{1} \pm T_{2}\right)
$$

where

$$
\begin{aligned}
T_{1} & =\left[\begin{array}{ccccc}
1 & \tau_{1} & \tau_{2} & \cdots & \tau_{i-1} \\
0 & 1 & \tau_{1} & \cdots & \tau_{i-2} \\
0 & 0 & 1 & \cdots & \tau_{i-3} \\
\cdots & \cdots & \cdots & \cdots & \cdots \\
0 & 0 & 0 & \cdots & 1
\end{array}\right], \\
T_{2} & =\left[\begin{array}{ccccc}
\tau_{n-i+1} & \tau_{n-i+2} & \cdots & \tau_{n-1} & \tau_{n} \\
\tau_{n-i+2} & \tau_{n-i+3} & \cdots & \tau_{n} & 0 \\
\cdots & \cdots & \cdots & \cdots & \cdots \\
\tau_{n-1} & \tau_{n} & \cdots & 0 & 0 \\
\tau_{n} & 0 & \cdots & 0 & 0
\end{array}\right] .
\end{aligned}
$$

Furthermore, the following conditions are satisfied:

(C1) We assume that $\mathbb{D}_{n-1}^{-}\left(\xi_{0}, u\right)=0, \mathbb{D}_{n-1}^{+}\left(\xi_{0}, u\right)>0, P_{\xi_{0}}(1)>0,(-1)^{n} P_{\xi_{0}}(-1)>0$, $\mathbb{D}_{i}^{ \pm}\left(\xi_{0}, u\right)>0$, for $i=n-3, n-5, \ldots, 2$ (or 1$)$, when $n$ is odd (or even), this is known as the eigenvalue criterion.

(C2) Next, we suppose that $\left(\frac{d}{d \xi}\left(\mathbb{D}_{n-1}^{-}(\xi, u)\right)\right)_{\xi=\xi_{0}} \neq 0$, and this is known as the transversality criterion for the Hopf bifurcation.

(C3) Finally, we consider that $\cos \left(\frac{2 \pi}{l}\right) \neq \varphi$, or the resonance condition $\cos \left(\frac{2 \pi}{l}\right)=\varphi$, where $l=3,4,5, \ldots$, and $\varphi=1-0.5 P_{\xi_{0}}(1) \mathbb{D}_{n-3}^{-}\left(\xi_{0}, u\right) / \mathbb{D}_{n-2}^{+}\left(\xi_{0}, u\right)$, which is called the nonresonance, or resonance criterion, then Hopf bifurcation takes place for the critical value $\xi_{0}$.

If we take $n=3$, then the following lemma provides us with the parametric conditions for which model (1.3) undergoes a Hopf bifurcation whenever $s$ is chosen as bifurcation parameter.

Lemma 3.2 The equilibrium point $E_{*}$ for model (1.3) undergoes a Hopf bifurcation at critical parametric value $s=s_{0}$ if the following conditions are satisfied:

$$
1-\gamma_{1}+\gamma_{0}\left(\gamma_{2}-\gamma_{0}\right)=0, \quad 1+\gamma_{1}-\gamma_{0}\left(\gamma_{2}+\gamma_{0}\right)>0,
$$




$$
\begin{aligned}
& 1+\gamma_{2}+\gamma_{1}+\gamma_{0}>0, \quad 1-\gamma_{2}+\gamma_{1}-\gamma_{0}>0, \\
& \frac{d}{d s}\left(1-\gamma_{1}+\gamma_{0}\left(\gamma_{2}-\gamma_{0}\right)\right)_{s=s_{0}} \neq 0,
\end{aligned}
$$

and

$$
\cos \left(\frac{2 \pi}{l}\right) \neq 1-\frac{1+\gamma_{2}+\gamma_{1}+\gamma_{0}}{2\left(1+\gamma_{0}\right)}, \quad l=3,4,5, \ldots
$$

where $\gamma_{2}, \gamma_{1}$, and $\gamma_{0}$ are listed in (2.7), and $s_{0}$ denotes a possible real root for $1-\gamma_{1}(s)+$ $\gamma_{0}(s)\left(\gamma_{2}(s)-\gamma_{0}(s)\right)=0$.

Proof By choosing $n=3$ and selecting $s$ as bifurcation parameter, it follows from Lemma 3.1 and Eq. (2.6) that

$$
\begin{aligned}
& \mathbb{D}_{2}^{-}(s)=1-\gamma_{1}+\gamma_{0}\left(\gamma_{2}-\gamma_{0}\right)=0, \quad \mathbb{D}_{2}^{+}(s)=1+\gamma_{1}-\gamma_{0}\left(\gamma_{2}+\gamma_{0}\right)>0, \\
& P_{s}(1)=1+\gamma_{2}+\gamma_{1}+\gamma_{0}>0, \quad(-1)^{3} P_{s}(-1)=1-\gamma_{2}+\gamma_{1}-\gamma_{0}>0 \\
& \left(\frac{d}{d s}\left(\mathbb{D}_{2}^{-}(s)\right)\right)_{s=s_{0}}=\frac{d}{d s}\left(1-\gamma_{1}+\gamma_{0}\left(\gamma_{2}-\gamma_{0}\right)\right)_{s=s_{0}} \neq 0,
\end{aligned}
$$

and

$$
1-0.5 P_{s}(1) \mathbb{D}_{0}^{-}(s) / \mathbb{D}_{1}^{+}(s)=1-\frac{1+\gamma_{2}+\gamma_{1}+\gamma_{0}}{2\left(1+\gamma_{0}\right)} .
$$

\section{Flip bifurcation analysis}

Now we investigate the parametric conditions for which a unique positive steady state for discrete-time system (1.3) encounters a period-doubling (flip) bifurcation. For such an investigation, we use an explicit criterion of the period-doubling (flip) bifurcation without computing the eigenvalues for the Jacobian matrix of a given model. For this purpose, we need the following result.

Lemma 4.1 ([20]) An n-dimensional system of discrete type is considered as follows: $Z_{k+1}=$ $F_{\xi}\left(Z_{k}\right)$ with similar conditions (3.1), (3.2) and (3.3) to those stated in Lemma 3.1. Next, it is assumed that the following criteria are satisfied:

$(H 1) P_{\xi_{0}}(-1)=0, \mathbb{D}_{n-1}^{ \pm}\left(\xi_{0}, u\right)>0, P_{\xi_{0}}(1)>0, \mathbb{D}_{i}^{ \pm}\left(\xi_{0}, u\right)>0, i=n-2, n-4, \ldots, 1$ (or 2$)$, when $n$ is even (or odd, respectively), and this is known as the eigenvalue criterion for flip bifurcation.

(H2) $\frac{\sum_{i=1}^{n}(-1)^{n-i} \tau_{i}^{\prime}}{\sum_{i=1}^{n}(-1)^{n-i}(n-i+1) \tau_{i-1}} \neq 0$, where $\tau_{i}^{\prime}$ denotes derivative of $\tau(\xi)$ at $\xi=\xi_{0}$, and this is called the transversality criterion, then a flip bifurcation occurs for the critical value $\xi_{0}$.

Furthermore, if we take $n=3$ in Lemma 4.1, then we obtain the following result for model (1.3) providing conditions for a flip bifurcation when $s$ is chosen as the bifurcation parameter.

Lemma 4.2 The equilibrium $E_{*}$ for model (1.3) undergoes a flip bifurcation at critical value $s=s_{0}$ if the following conditions are satisfied:

$$
1-\gamma_{1}+\gamma_{0}\left(\gamma_{2}-\gamma_{0}\right)>0, \quad 1+\gamma_{1}-\gamma_{0}\left(\gamma_{2}+\gamma_{0}\right)>0
$$




$$
1+\gamma_{2}+\gamma_{1}+\gamma_{0}>0, \quad 1-\gamma_{2}+\gamma_{1}-\gamma_{0}=0, \quad 1 \pm \gamma_{0}>0
$$

and

$$
\frac{\gamma_{2^{\prime}}-\gamma_{1}^{\prime}+\gamma_{0}^{\prime}}{3-2 \gamma_{2}+\gamma_{1}} \neq 0,
$$

where $\gamma_{2}, \gamma_{1}$, and $\gamma_{0}$ are written in (2.7), $\gamma_{i}^{\prime}$ is derivative of $\gamma(s)$ at the critical value $s=s_{0}$, and $s_{0}$ represents a possible real root for $1-\gamma_{2}(s)+\gamma_{1}(s)-\gamma_{0}(s)=0$.

\section{Chaos control}

Next, we investigate a chaos control technique for the discrete-time model (1.3). Keeping in view the simplicity, a hybrid control methodology is chosen which was firstly proposed by Luo et al. [21]. The hybrid chaos control method consists of a single control parameter which lies inside the open unit interval. Furthermore, the implementation for such a hybrid control strategy is comparatively simple one and it is based on both parameter perturbation and state feedback control strategy. It is worthwhile to mention some other investigations for controlling chaos in discrete-time systems and the interested reader is referred to [22-31].

One can apply a hybrid control method to obtain a corresponding control model of the following type:

$$
\left\{\begin{array}{l}
x_{n+1}=B x_{n} \exp \left(s\left(1-\frac{x_{n}+y_{n}}{k}\right)-\frac{\alpha y_{n}}{c+y_{n}}\right)+(1-B) x_{n} \\
y_{n+1}=B y_{n} \exp \left(\frac{\alpha x_{n}}{c+y_{n}}-\frac{\beta z_{n}}{y_{n}+\delta}-\mu\right)+(1-B) y_{n} \\
z_{n+1}=B z_{n} \exp \left(\frac{\beta \gamma y_{n}}{y_{n}+\delta}-\eta\right)+(1-B) z_{n}
\end{array}\right.
$$

where $0<B<1$ is used for the controlling parameter. Moreover, the variational matrix for model (5.1) evaluated at its positive steady state $\left(x^{*}, y^{*}, z^{*}\right)$ is calculated as follows:

$$
\left(\begin{array}{ccc}
1-\frac{B s x^{*}}{k} & B a_{12} & 0 \\
\frac{B \alpha \delta \eta}{\beta \gamma-\eta+c \delta \eta} & 1+B a_{22} & -\frac{B \eta}{\gamma} \\
0 & B a_{32} & 1
\end{array}\right)
$$

where $a_{12}, a_{22}$ and $a_{32}$ are listed in (2.5). Then the characteristic polynomial equation for the aforementioned variational matrix of controlled model (5.1) is calculated as follows:

$$
P_{3}(\lambda)=\lambda^{3}+\delta_{2} \lambda^{2}+\delta_{1} \lambda+\delta_{0}=0,
$$

where

$$
\left\{\begin{array}{l}
\delta_{2}=-3-B a_{22}+\frac{B s x^{*}}{k} \\
\delta_{1}=3-\frac{B^{2} \alpha \delta \eta a_{12}}{\beta \gamma-\eta+c \delta \eta}+2 B a_{22}+\frac{B^{2} \eta a_{32}}{\gamma}-\frac{2 B s x^{*}}{k}-\frac{B^{2} s a_{22} x^{*}}{k} \\
\delta_{0}=-1+\frac{B^{2} \alpha \delta \eta a_{12}}{\beta \gamma-\eta+c \delta \eta}-B a_{22}-\frac{B^{2} \eta a_{32}}{\gamma}+\frac{B s x^{*}}{k}+\frac{B^{2} s a_{22} x^{*}}{k}+\frac{B^{3} s \eta a_{32} x^{*}}{k \gamma}
\end{array}\right.
$$

Then it is obvious that the equilibrium point for model (5.1) is a sink as long as the regulator poles are of modulus less than 1 . Such parametric conditions are stated in the following lemma. 
Lemma 5.1 The equilibrium $E_{*}$ for model (5.1) is a sink and locally asymptotically stable if and only if the following inequalities are satisfied:

$$
\left|\delta_{2}+\delta_{0}\right|<1+\delta_{1}, \quad\left|\delta_{2}-3 \delta_{0}\right|<3-\delta_{1}, \quad \text { and } \quad \delta_{0}^{2}+\delta_{1}-\delta_{0} \delta_{2}<1
$$

\section{Numerical simulation and discussion}

Example 6.1 First of all, we select the parametric values as follows: $\alpha=0.5, k=6.2, \beta=0.9$, $\delta=0.85, \mu=0.5, \gamma=1.5, \eta=0.1, c=8.5$ and $s \in[1.8,2.8]$. Moreover, the initial values are taken as $\left(x_{0}, y_{0}, z_{0}\right)=(6.06,0.068,1.45)$, then it is observed that system (1.3) undergoes period-doubling (flip) bifurcation as we choose $s=2.09247$. Furthermore, it is easy to see that, for the selected parametric values $\alpha=0.5, k=6.2, \beta=0.9, \delta=0.85, \mu=0.5, \gamma=$ $1.5, \eta=0.1, c=8.5$ and $s=2.09247$, one can easily calculate the characteristic polynomial equation for model (1.3):

$$
\lambda^{3}-0.35314 \lambda^{2}-0.865105 \lambda+0.488035=0 .
$$

Furthermore, the roots for Eq. (6.1) are calculated as $\lambda_{1,2}=0.67657 \pm 0.174034 i$, and $\lambda_{3}=-1$, so that we have $\left|\lambda_{1,2}\right|=0.698595$. Consequently, the eigenvalues condition flip bifurcation is justified. Obviously, all conditions in Lemma 4.2 are listed and justified:

$$
\begin{aligned}
& 1-\gamma_{1}+\gamma_{0}\left(\gamma_{2}-\gamma_{0}\right)=1.45458>0, \\
& 1+\gamma_{1}-\gamma_{0}\left(\gamma_{2}+\gamma_{0}\right)=0.0690615>0, \\
& 1+\gamma_{2}+\gamma_{1}+\gamma_{0}=0.26979>0, \quad 1-\gamma_{2}+\gamma_{1}-\gamma_{0}=0, \\
& 1+\gamma_{0}=1.48803>0, \quad 1-\gamma_{0}=0.511965>0,
\end{aligned}
$$

and

$$
\frac{\gamma_{2^{\prime}}-\gamma_{1}^{\prime}+\gamma_{0}^{\prime}}{3-2 \gamma_{2}+\gamma_{1}}=1.00616 \neq 0
$$

Furthermore, diagrams related to bifurcation and maximum Lyapunov exponents (MLEs) are presented in Fig. 1.

We select parameters $\alpha=0.5, k=6.2, \beta=0.9, \delta=0.85, \mu=0.5, \gamma=1.5, \eta=0.1, c=$ 8.5 and $s=2.8$ to demonstrate the effectiveness of chaos control strategy given in system (5.1). For these selected parametric values model (1.3) has a positive steady state given by $\left(x^{*}, y^{*}, z^{*}\right)=(6.08429,0.068,1.45641)$. On the other hand, the variational matrix of model $(5.1)$ at the steady state $\left(x^{*}, y^{*}, z^{*}\right)=(6.08429,0.068,1.45641)$ is computed as follows:

$$
\left(\begin{array}{ccc}
1-2.74774 B & -3.96945 B & 0 \\
0.0215463 B & 1-0.600378 B & -0.0666667 B \\
0 & 1.98312 B & 1
\end{array}\right) .
$$

An application of the Jury condition shows that the steady state for controlled model (5.1) is a sink if and only if $0<B<0.738529$, and consequently the length for the stability interval is given by 0.738529 . This shows that the length of the stability interval is comparatively suitable although the value $s=2.8$ is selected from the extreme right end of the chaotic interval. 


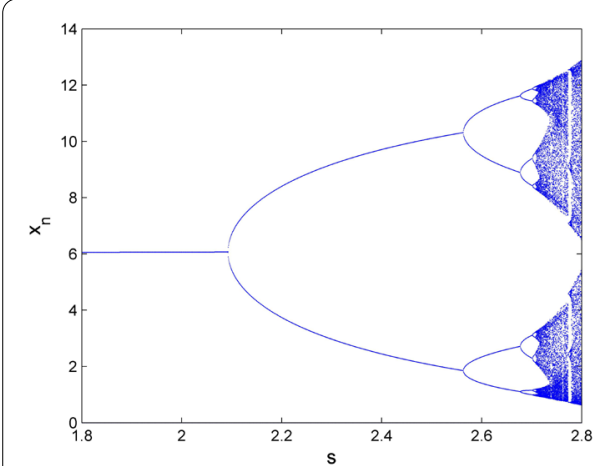

(a) Bifurcation diagram for $x_{n}$

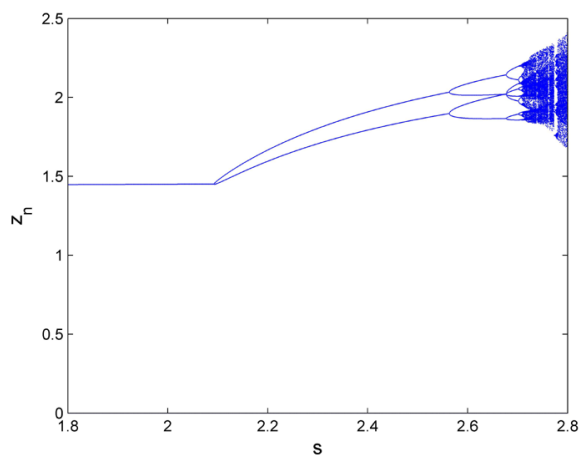

(c) Bifurcation diagram for $z_{n}$

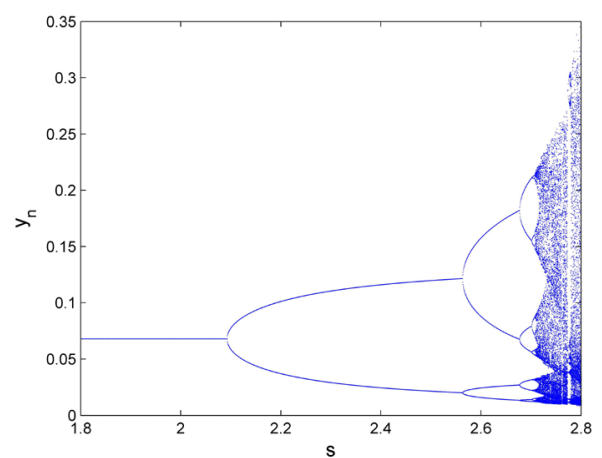

(b) Bifurcation diagram for $y_{n}$

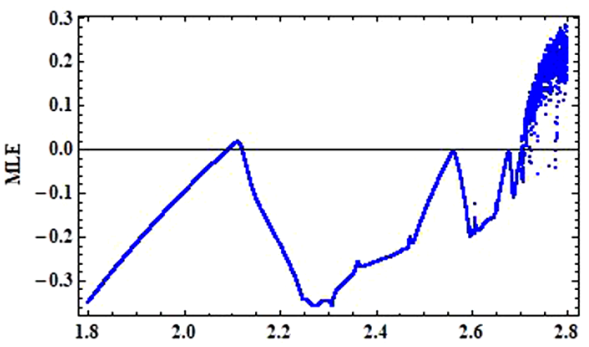

(d) Maximum Lyapunov exponents

Figure 1 Bifurcation diagrams and MLE for system (1.3) with $\alpha=0.5, k=6.2, \beta=0.9, \delta=0.85, \mu=0.5$, $\gamma=1.5, \eta=0.1, c=8.5, s \in[1.8,2.8]$ and initial conditions $\left(x_{0}, y_{0}, z_{0}\right)=(6.06,0.068,1.45)$

Example 6.2 Next, we choose the parametric and initial values as follows: $\alpha=1.6, k=1.4$, $\beta=0.13, \delta=0.4, \mu=0.15, \gamma=2.95, \eta=0.1, c=0.5,\left(x_{0}, y_{0}, z_{0}\right)=(1.10514,0.141,6.2505)$ and bifurcation parameter $s \in[1.8,2.8]$. In this case, it is observed that for these selected values system (1.3) undergoes a Hopf bifurcation at $s=1.93866$, and bifurcation diagrames are depicted in Fig. 2. Furthermore, if we take values as $\alpha=1.6, k=1.4, \beta=0.13, \delta=0.4$, $\mu=0.15, \gamma=2.95, \eta=0.1, c=0.5$ and $s=1.93866$, then the characteristic polynomial equation for model (1.3) is calculated, thus:

$$
\lambda^{3}-1.75076 \lambda^{2}+0.8277 \lambda+0.0934286=0 .
$$

Keeping in view the above selected values the roots for the aforementioned characteristic equation are given by $\lambda_{1}=-0.0934286$, and $\lambda_{2,3}=0.922095 \pm 0.386964 i$ so that the modulus for complex conjugate roots is $\left|\lambda_{2,3}\right|=1$. Consequently, the eigenvalues condition for Hopf bifurcation is justified. On the other hand, all other conditions in Lemma 3.2 are verified:

$$
\begin{aligned}
& \mathbb{D}_{2}^{-}(1.93866)=1-\gamma_{1}+\gamma_{0}\left(\gamma_{2}-\gamma_{0}\right)=0, \\
& \mathbb{D}_{2}^{+}(1.93866)=1+\gamma_{1}-\gamma_{0}\left(\gamma_{2}+\gamma_{0}\right)=1.98254>0, \\
& P_{1.93866}(1)=1+\gamma_{2}+\gamma_{1}+\gamma_{0}=0.170367>0, \\
& (-1)^{3} P_{1.93866}(-1)=1-\gamma_{2}+\gamma_{1}-\gamma_{0}=3.48503>0,
\end{aligned}
$$




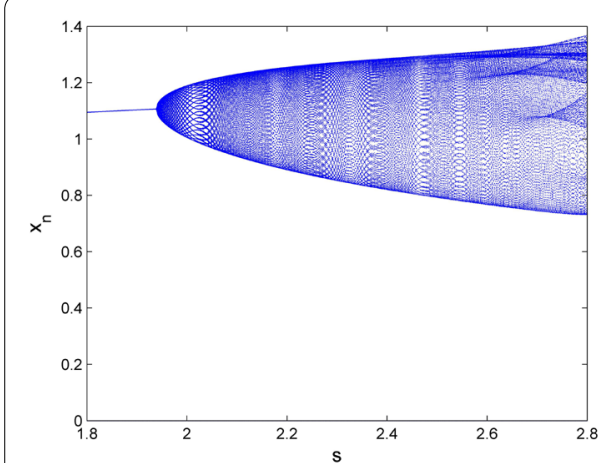

(a) Bifurcation diagram for $x_{n}$

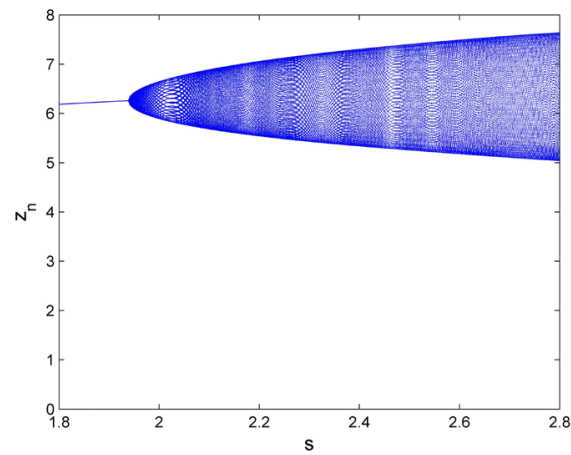

(c) Bifurcation diagram for $z_{n}$

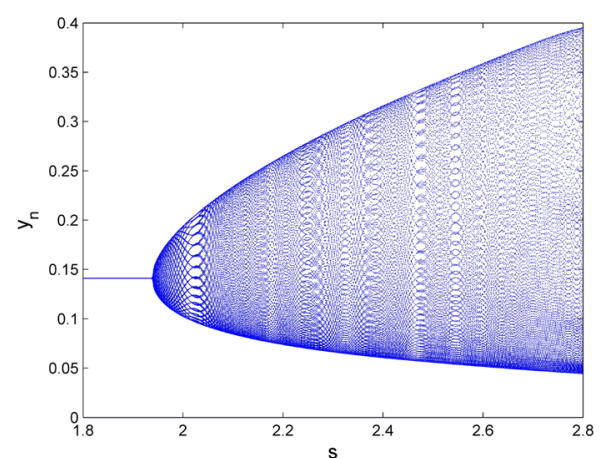

(b) Bifurcation diagram for $y_{n}$

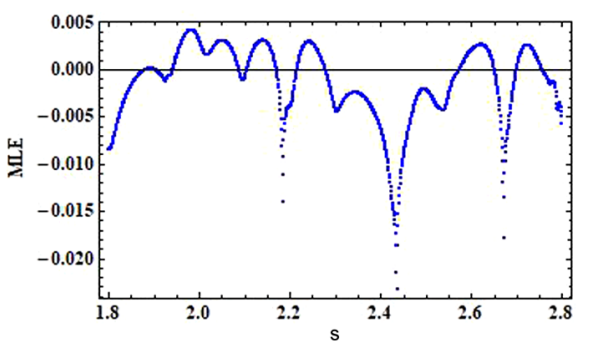

(d) Maximum Lyapunov exponents

Figure 2 Bifurcation diagrams and MLE for system (1.3) with $\alpha=1.6, k=1.4, \beta=0.13, \delta=0.4, \mu=0.15$, $\gamma=2.95, \eta=0.1, c=0.5,\left(x_{0}, y_{0}, z_{0}\right)=(1.10514,0.141,6.2505)$ and $s \in[1.8,2.8]$

$$
\left(\frac{d}{d s}\left(\mathbb{D}_{2}^{-}(s)\right)\right)_{s=1.93866}=\frac{d}{d s}\left(1-\gamma_{1}+\gamma_{0}\left(\gamma_{2}-\gamma_{0}\right)\right)_{s=1.93866}=-0.146935 \neq 0,
$$

and

$$
1-0.5 P_{1.93866}(1) \mathbb{D}_{0}^{-}(1.93866) / \mathbb{D}_{1}^{+}(1.93866)=1-\frac{1+\gamma_{2}+\gamma_{1}+\gamma_{0}}{2\left(1+\gamma_{0}\right)}=0.922095
$$

By considering the equation $\cos \left(\frac{2 \pi}{l}\right)=0.922095$, we obtain $l= \pm 15.8133$. Therefore, the condition for non-resonance is also correctly justified. Furthermore, some phase portraits for various values of $s$ are depicted in Fig. 3. Moreover, in Fig. 3(b) the appearance of closed invariant circle at $s=1.93866$ is clearly observed.

Furthermore, we choose values as $\alpha=1.6, k=1.4, \beta=0.13, \delta=0.4, \mu=0.15, \gamma=$ 2.95, $\eta=0.1, c=0.5$ and $s=2.8$ in order to show the effectiveness for hybrid control method implemented in model (5.1). With these selected values, one can easily obtain the following variational matrix for model (5.1) computed at its positive steady state (1.15347, 0.141093, 6.55112):

$$
\left(\begin{array}{ccc}
1-2.30694 B & -3.91727 B & 0 \\
0.210873 B & 1+0.296809 B & -0.0338983 B \\
0 & 3.43239 B & 1
\end{array}\right) \text {. }
$$




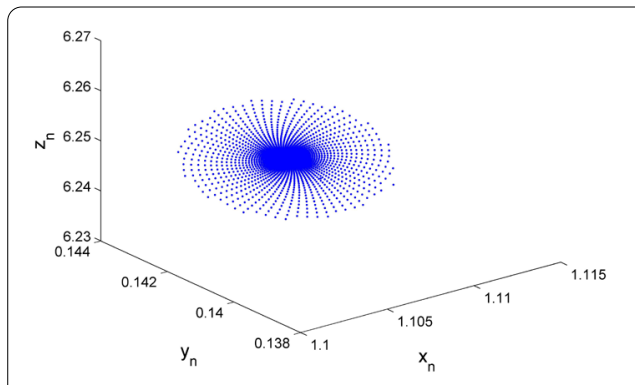

(a) Phase portrait at $s=1.92$

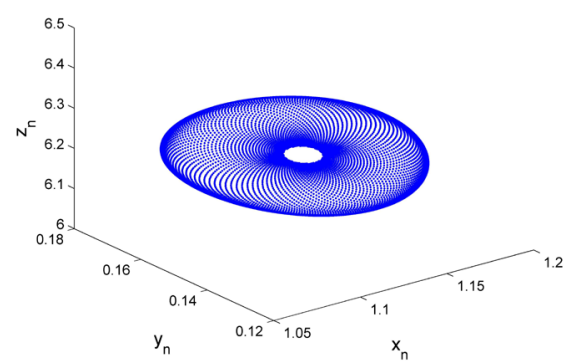

(c) Phase portrait at $s=1.95$

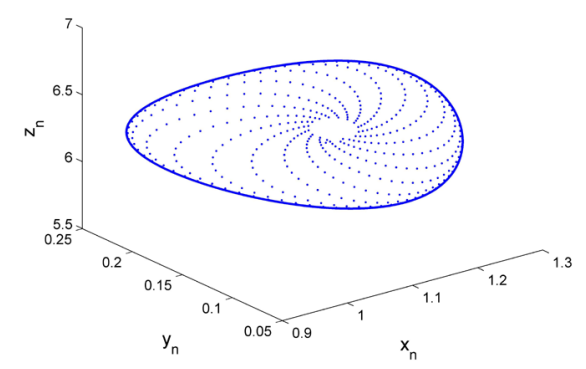

(e) Phase portrait at $s=2.1$

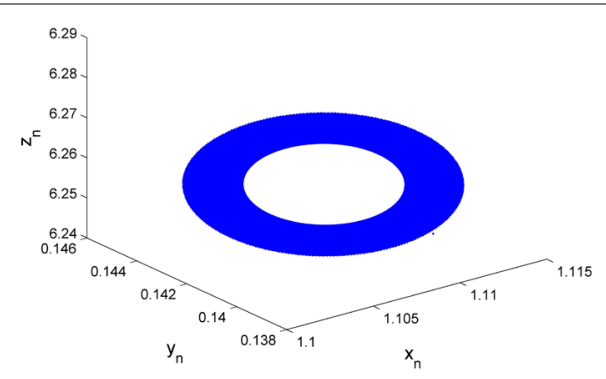

(b) Phase portrait at $s=1.93866$

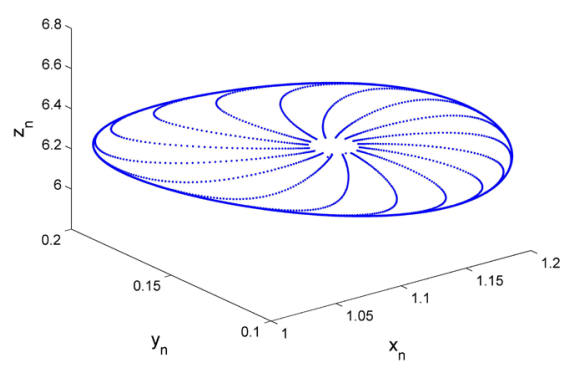

(d) Phase portrait at $s=2$

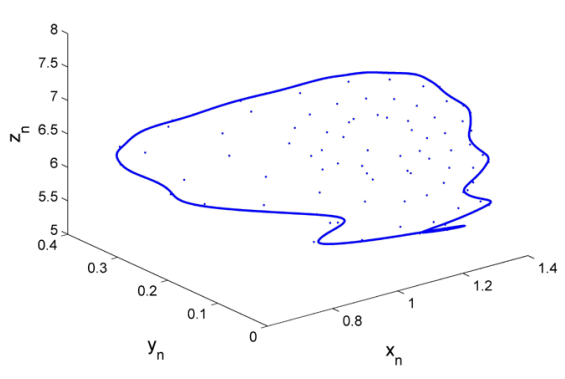

(f) Phase portrait at $s=2.8$

Figure 3 Phase portraits of system (1.3) with various values of $s$

An application of the Jury condition shows that the steady state for controlled model (5.1) is a sink if and only if $0<B<0.446796$. Consequently, in this case of a Hopf bifurcation, the length for the stability interval is less than as that of flip bifurcation.

Example 6.3 Finally, in order to select the parametric values in a range observed in an actual predator-prey system, we choose most of our basic parametric values from the study of eco-epidemiological models of pelicans at risk in the Salton Sea by Chattopadhyay et al. $[7,9]$. These parametric values are given in Table 1 . Keeping in view the tabulated values in Table 1, we choose $c=1.2$ as the crowding effect in the contact rate and $s \in[1.5,3]$ as the bifurcation parameter. Then the positive equilibrium of system (1.3) undergoes a perioddoubling bifurcation at $s=2.00701$. The bifurcation diagrams and MLE are depicted in Fig. 4.

\section{Concluding remarks}

In this paper, the qualitative nature of discrete-time system (1.3) is investigated. The discrete-time system is obtained with implementation of a piecewise constant argument 
Table 1 Estimation for values of the parameters

\begin{tabular}{lll}
\hline Parameter & Value & Source \\
\hline$k$ & 40.0 tonnes & {$[7]$} \\
$\alpha$ & 0.006 per day & {$[7,9]$} \\
$\beta$ & 14.5 per day & {$[7]$} \\
$\delta$ & 14.0 tonnes & {$[7]$} \\
$\mu$ & 0.0019 per day & {$[7]$} \\
$\gamma$ & 0.765517 per day & {$[7]$} \\
$\eta$ & 0.09 per day & {$[9]$} \\
\hline
\end{tabular}

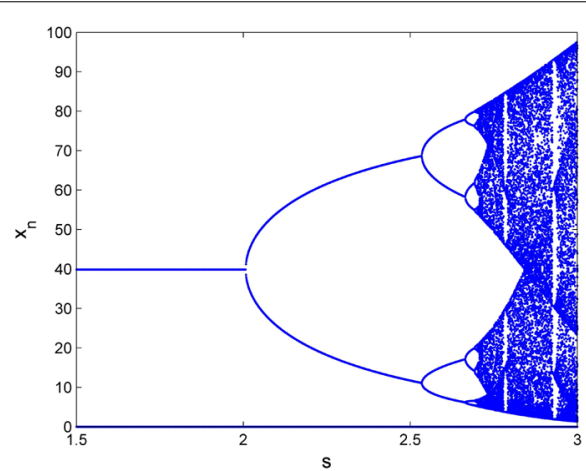

(a) Bifurcation diagram for $x_{n}$

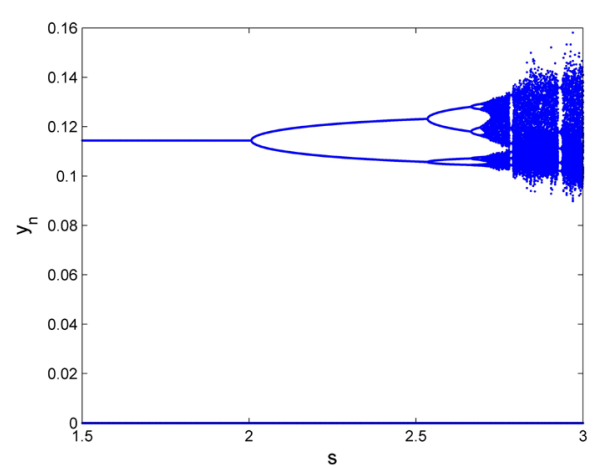

(b) Bifurcation diagram for $y_{n}$

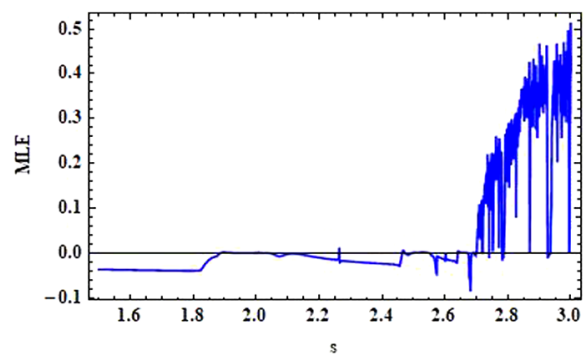

(c) Maximum Lyapunov exponents

Figure 4 Bifurcation diagrams and MLE for system (1.3) with $\alpha=0.006, k=40, \beta=14.5, \delta=14, \mu=0.0019$, $\gamma=0.765517, \eta=0.09, c=1.2,\left(x_{0}, y_{0}, z_{0}\right)=(39.8735,0.114441,0.20291)$ and $s \in[1.5,3]$

and bifurcating and chaotic behavior is studied with the help of standard mathematical techniques. The local behavior for equilibria is investigated by implementing the method of linearization. Furthermore, explicit criteria for Neimark-Sacker bifurcation and perioddoubling bifurcation are used for the investigation of the bifurcating behavior about positive equilibrium point. On the other hand, parametric values are also selected in a range observed in an actual predator-prey system, and in this case there is a brighter chance of occurrence of period-doubling bifurcation in the discrete-time model. A hybrid control strategy based on parameter perturbation and state feedback method is used for controlling the fluctuating and chaotic behavior of model (1.3). Moreover, it is noticed that the hybrid chaos control method can stabilize the chaotic orbits more effectively due to the emergence of a period-doubling (flip) bifurcation as compared to that of a Neimark-Sacker (Hopf) bifurcation. Furthermore, it is investigated that the parameter $c$, representing the crowding effect in the system, is more appropriate for the emergence of period-doubling (flip) and Neimark-Sacker (Hopf) bifurcations whenever it is varied in some suitable in- 
terval. It is examined that larger values of $c$ may result flip bifurcation, meanwhile smaller values for $c$ may result from a Neimark-Sacker bifurcation.

\author{
Acknowledgements \\ The authors are grateful to the referees for their excellent suggestions, which greatly improved the presentation of the \\ paper.
}

\title{
Funding
}

Not applicable.

Availability of data and materials

Not applicable.

\section{Competing interests}

It is declared that none of the authors have any competing interests in this manuscript.

\section{Authors' contributions}

All authors contributed equally to the writing of this paper. All authors read and approved the final manuscript.

\section{Author details}

${ }^{1}$ Department of Mathematics, University of Azad Jammu and Kashmir, Muzaffarabad, Pakistan. ${ }^{2}$ Department of Mathematics, University of Poonch Rawalakot, Azad Kashmir, Pakistan.

\section{Publisher's Note}

Springer Nature remains neutral with regard to jurisdictional claims in published maps and institutional affiliations.

Received: 12 July 2018 Accepted: 15 January 2019 Published online: 25 January 2019

\section{References}

1. Hadeler, K.P., Freedman, H.I.: Predator-prey population with parasite infection. J. Math. Biol. 27,609-631 (1989)

2. Freedman, H.I.: A model of predator-prey dynamics as modified by the action of parasite. Math. Biosci. 99, 143-155 (1990)

3. Beltrami, E., Carroll, T.O.: Modelling the role of viral disease in recurrent phytoplankton blooms. J. Math. Biol. 32, 857-863 (1994)

4. Venturino, E.: Epidemics in predator-prey models: disease in the prey. In: Arino, O., Axelrod, D., Kimmel, M., Langlais, M. (eds.) Mathematical Population Dynamics: Analysis of Heterogeneity, vol. 1, pp. 381-393 (1995)

5. Chattopadhyay, J., Arino, O.: A predator-prey model with disease in the prey. Nonlinear Anal. 36, 747-766 (1999)

6. Chattopadhyay, J., Ghosal, G., Chaudhuri, K.C.: Nonselective harvesting of a prey-predator community with infected prey. Korean J. Comput. Appl. Math. 6, 601-616 (1999)

7. Chattopadhyay, J., Bairagi, N.: Pelicans at risk in Salton Sea-an eco-epidemiological model. Ecol. Model. 136, $103-112(2001)$

8. Xiao, Y., Chen, L.: A ratio-dependent predator-prey model with disease in the prey. Appl. Math. Comput. 131(2), 397-414 (2002)

9. Chattopadhyay, J., Srinivasu, P.D.N., Bairagi, N.: Pelicans at risk in Salton Sea-an eco-epidemiological model-II. Ecol. Model. 167, 199-211 (2003)

10. Pal, S., Kundu, K., Chattopadhyay, J.: Role of standard incidence in an eco-epidemiological system: a mathematical study. Ecol. Model. 199(3), 229-239 (2006)

11. Bairagi, N., Roy, P.K., Chattopadhyay, J.: Role of infection on the stability of a predator-prey system with several response functions - a comparative study. J. Theor. Biol. 248, 10-25 (2007)

12. Bairagi, N., Chaudhury, S., Chattopadhyay, J.: Harvesting as a disease control measure in an eco-epidemiological system - a theoretical study. Math. Biosci. 217, 134-144 (2009)

13. Wikipedia contributors, Salton Sea, https://en.wikipedia.org/w/index.php?title=Salton_Sea oldid=867807799

14. Rocke, T., Converse, K., Meteyer, C., McLean, B.: The impact of disease in the American white pelican in North America. BioOne 28, 87-94 (2005)

15. Kundu, K., Chattopadhyay, J.: A ratio-dependent eco-epidemiological model of the Salton Sea. Math. Methods Appl. Sci. 29, 191-207 (2006)

16. Camouzis, E., Ladas, G.: Dynamics of Third-Order Rational Difference Equations with Open Problems and Conjectures. Chapman \& Hall, New York (2008)

17. Din, Q., Elsadany, A.A., Khalil, H.: Neimark-Sacker bifurcation and chaos control in a fractional-order plant-herbivore model. Discrete Dyn. Nat. Soc. 2017, 1-15 (2017)

18. Matouk, A.E., Elsadany, A.A., Ahmed, E., Agiza, H.N.: Dynamical behavior of fractional-order Hastings-Powell food chain model and its discretization. Commun. Nonlinear Sci. Numer. Simul. 27, 153-167 (2015)

19. Wen, G.: Criterion to identify Hopf bifurcations in maps of arbitrary dimension. Phys. Rev. E 72, Article ID 026201 (2005)

20. Wen, G., Chen, S., Jin, Q.: A new criterion of period-doubling bifurcation in maps and its application to an inertial impact shaker. J. Sound Vib. 311, 212-223 (2008)

21. Luo, X.S., Chen, G.R., Wang, B.H.: Hybrid control of period-doubling bifurcation and chaos in discrete nonlinear dynamical systems. Chaos Solitons Fractals 18, 775-783 (2003) 
22. Din, Q: Complexity and chaos control in a discrete-time prey-predator model. Commun. Nonlinear Sci. Numer. Simul. 49, 113-134 (2017)

23. Din, Q.: Bifurcation analysis and chaos control in discrete-time glycolysis models. J. Math. Chem. 56(3), 904-931 (2018)

24. Din, Q., Donchev, T., Kolev, D.: Stability, bifurcation analysis and chaos control in chlorine dioxide-iodine-malonic acid reaction. MATCH Commun. Math. Comput. Chem. 79(3), 577-606 (2018)

25. Din, Q.: Neimark-Sacker bifurcation and chaos control in Hassell-Varley model. J. Differ. Equ. Appl. 23(4), 741-762 (2017)

26. Din, Q., Gümüş, Ö.A., Khalil, H.: Neimark-Sacker bifurcation and chaotic behaviour of a modified host-parasitoid model. Z. Naturforsch. A 72(1), 25-37 (2017)

27. Din, Q.: Controlling chaos in a discrete-time prey-predator model with Allee effects. Int. J. Dyn. Control 6(2), 858-872 (2018)

28. Din, Q.: Qualitative analysis and chaos control in a density-dependent host-parasitoid system. Int. J. Dyn. Control 6(2), 778-798 (2018)

29. Din, Q:: A novel chaos control strategy for discrete-time Brusselator models. J. Math. Chem. 56(10), 3045-3075 (2018)

30. Din, Q.: Stability, bifurcation analysis and chaos control for a predator-prey system. J. Vib. Control (2018). https://doi.org/10.1177/1077546318790871

31. Din, Q., lqbal, M.A.: Bifurcation analysis and chaos control for a discrete-time enzyme model. Z. Naturforsch. A (2018). https://doi.org/10.1515/zna-2018-0254

\section{Submit your manuscript to a SpringerOpen ${ }^{\circ}$ journal and benefit from:}

- Convenient online submission

- Rigorous peer review

- Open access: articles freely available online

- High visibility within the field

- Retaining the copyright to your article

Submit your next manuscript at $\gg$ springeropen.com 